Mitchison, D. A. (1951). J. gen. Microbiol. 5, 596-604.

\title{
The Segregation of Streptomycin-Resistant Variants of Mycobacterium tuberculosis into Groups with Characteristic Levels of Resistance
}

\author{
By D. A. MITCHISON \\ Department of Bacteriology, Postgraduate Medical School of London
}

SUMMARY: Three initially streptomycin-sensitive strains of Mycobacterium tuberculosis, two from patients with tuberculosis, and one the laboratory strain $\mathrm{H3} \mathbf{R v}$, were grown for 6 weeks on Herrold's agar medium containing graded concentrations of streptomycin. Discrete colonies of $M$. tuberculosis that grew on the streptomycin media were tested for streptomycin sensitivity in Tween 80 albumin medium.

In all three strains the distribution of resistance was discontinuous, the subcultures from the colonies being either sensitive, or resistant to streptomycin in three distinct ranges of minimal inhibitory concentrations, namely, 0.25-0.5, 1-2 and 32-64 $\mu \mathrm{g} . / \mathrm{ml}$.; and of initial populations of $0.4-1.0 \times 10^{\circ}$ bacilli, about 1000 were in the first, slightly resistant group, about 100 in the second, and about 2 were in the third, highly resistant group.

Of the two patients from whom the two of the strains were isolated, one developed a highly resistant strain in his sputum during treatment, whereas the other developed a strain only of slight resistance, in spite of prolonged streptomycin treatment.

The occurrence of streptomycin-resistant variants of $M$. tuberculosis in populations of organisms which have not previously been exposed to the antibiotic has been demonstrated in laboratory strains by Vennesland, Ebert \& Bloch (1947) and Yegian \& Vanderlinde (1948), and in strains obtained directly from patients by Pyle (1947). Yegian \& Vanderlinde inoculated plates of a medium containing the detergent Tween 80 and streptomycin with large numbers of bacilli, and found that the number of resistant colonies that grew depended on the concentration of streptomycin. The number of colonies decreased markedly when the concentration was increased from 1 to $10 \mu \mathrm{g} . / \mathrm{ml}$. and then to $100 \mu \mathrm{g} . / \mathrm{ml}$. With the higher concentrations, such as 100 and $1000 \mu \mathrm{g} . / \mathrm{ml}$., there was no significant difference in the number of colonies. Strains derived from colonies growing in the presence of $100 \mu \mathrm{g}$. $/ \mathrm{ml}$. were also capable of growth in $1000 \mu \mathrm{g} . / \mathrm{ml}$. One of these highly resistant variants was found in every $10^{8}-10^{9}$ bacilli in the original inoculum, though the proportions varied considerably with different strains and in different experiments with the same strain. Strains derived from colonies growing in the presence of $1 \mu \mathrm{g} . / \mathrm{ml}$. contained a larger proportion of highly resistant variants than did the original parent population. Meads \& Haslam (1949), investigating the occurrence of streptomycin-resistant variants of Klebsiella pneumoniae, found that the predominantly sensitive population contained two groups of variants, a small number of organisms slightly resistant to streptomycin and a much smaller number of highly resistant organisms. In their experiments slightly resistant strains contained the same proportion of highly resistant organisms as did the parent population, namely, $0 \cdot 5-4 \cdot 6$ per $10^{10}$ organisms in the inoculum. 
In a previous publication (Mitchison, 1949), sensitivity tests on 398 streptomycin-resistant strains of $\boldsymbol{M}$. tuberculosis, isolated from patients during their treatment with the antibiotic, were classified according to the concentration of streptomycin which inhibited their growth. There were three distinct groups of strains, each with a characteristic degree of resistance. In the first group strains were inhibited by 1-2 $\mu \mathrm{g} . / \mathrm{ml}$. and were about four times less sensitive than the standard sensitive strain $\mathrm{H37Rv}$; in the second, by $32-64 \mu \mathrm{g} . / \mathrm{ml}$; in the third group strains grew in at least $100 \mu \mathrm{g} . / \mathrm{ml}$. If we accept the view that the occurrence of resistant strains in the sputa of patients receiving streptomycin is due to the selection of the variants present in small numbers in the pre-treatment population of bacilli, then there are two possible explanations of the occurrence of these groups. Either all degrees of streptomycin resistance are to be found among these variants and the occurrence of the groups among the strains isolated from the patients are due to selective factors in the environment such as the maximum or minimum levels of streptomycin in the lesions; or the variants themselves tend to occur in these groups before the selective action of the presence of streptomycin in the lesions begins. An attempt has been made in this paper to establish whether the distribution of streptomycin resistance among variants occurring in a normal sensitive population is in fact discontinuous and whether the groups of variants with different resistances correspond with these found among strains isolated from treated patients.

\section{METHODS}

\section{Strains of Mycobacterium tuberculosis}

Three strains of tubercle bacilli of human type were used:

(1) H37 Rv, a virulent laboratory strain from which variants with a high degree of streptomycin resistance have been obtained.

(2) $\mathrm{Pa}$, a strain isolated from the sputum of a patient with pulmonary tuberculosis before treatment with streptomycin and preserved by drying in gelatin-ascorbate by the method of Stamp (1947). The patient received $1 \mathrm{~g}$. of streptomycin per day over a period of 6 months. Numerous cultures isolated from the sputum from the sixth week onwards yielded strains only two to four times less sensitive than the standard strain $\mathbf{H 3 7} \mathbf{R v}$.

(3) Pr, a strain isolated from a patient with a similar pulmonary condition before treatment was started with the same dosage of streptomycin over the same period of time. This culture was also preserved by drying. During treatment cultures from this patient yielded several strains capable of growth in $1000 \mu \mathrm{g}$. streptomycin $/ \mathrm{ml}$.

Streptomycin. The streptomycin used was the calcium chloride complex prepared by Merck and Co. (Rahway, N.J., U.S.A.).

Medium. Batches of Herrold's egg agar medium were prepared with the following composition: Lemco meat extract (Oxo Ltd., London), 3 g.; peptone, 10 g.; sodium chloride, 5 g.; glycerol, $50 \mathrm{ml}$.; agar, 15 g.; and distilled water to $1000 \mathrm{ml}$.

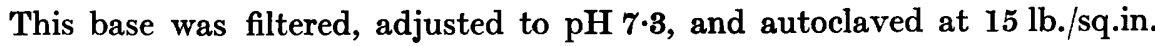


for $20 \mathrm{~min}$. Before use it was melted, cooled to $55-60^{\circ}$ and the yolk of an egg, separated with sterile precautions, was added to each $150 \mathrm{ml}$. The total amount of the medium required for each experiment was prepared in one batch. From this, appropriate quantities were transferred to a series of conical flasks containing streptomycin so that final streptomycin concentrations of $0,1,2,4, \ldots$, $1024 \mu \mathrm{g} . / \mathrm{ml}$. were obtained. The contents of each flask was distributed in $4 \mathrm{ml}$. lots to $3 \times 1$ in. flat-bottomed tubes plugged with cotton-wool and was allowed to set so that a flat plaque was left covering the bottom of the tube. The tubes were dried for about $4 \mathrm{hr}$. at $37^{\circ}$.

Table 1. The design of the test for growth in the presence of streptomycin

(The figures indicate the number of tubes of Herrold's medium inoculated with strains Pa.)

\begin{tabular}{|c|c|c|c|c|c|c|c|c|c|c|c|c|}
\hline \multirow{2}{*}{$\begin{array}{l}\text { Culture } \\
\text { dilution }\end{array}$} & \multicolumn{12}{|c|}{ Concentration of streptomycin in Herrold's medium $(\mu \mathrm{g} . / \mathrm{ml})}$. \\
\hline & $\mathbf{0}$ & 1 & $\mathbf{2}$ & 4 & 8 & $\begin{array}{c}16 \\
\text { No. } 0\end{array}$ & $\begin{array}{l}32 \\
\text { tube }\end{array}$ & 64 & 128 & 256 & 512 & 1024 \\
\hline $10^{0}$ & . & . & & 6 & 8 & 10 & 10 & 10 & 12 & 16 & 16 & 16 \\
\hline $10^{-1}$ & $\dot{.}$ & $\dot{6}$ & $\dot{6}$ & 6 & 6 & 6 & 6 & 6 & 6 & 6 & 6 & 6 \\
\hline $10^{-2}$ & . & 6 & 6 & 6 & 6 & . & . & . & . & . & . & . \\
\hline $10^{-8}$ & . & 6 & 6 & 6 & 6 & . & . & . & . & . & . & . \\
\hline $10^{-4}$ & . & 6 & 6 & 6 & 6 & . & . & . & . & . & . & . \\
\hline $10^{-5}$ & 6 & 6 & 6 & 6 & . & . & . & . & . & . & . & . \\
\hline $10^{-6}$ & 6 & 6 & 6 & 6 & . & . & . & . & . & . & . & . \\
\hline $10^{-7}$ & $\boldsymbol{6}$ & B & $\boldsymbol{B}$ & . & . & . & . & . & . & . & . & . \\
\hline $10^{-8}$ & 6 & 6 & 6 & . & . & . & . & . & . & . & . & . \\
\hline
\end{tabular}

Procedure. Roux bottles containing $200 \mathrm{ml}$. of Tween 80 albumin medium (Medical Research Council, 1948) were inoculated with the strain under investigation and were incubated at $37^{\circ}$ for 14 days. The contents of three bottles $(600 \mathrm{ml}$.) was centrifuged for 50-60 min. at 3000 r.p.m., and the deposit resuspended in $30-40 \mathrm{ml}$. of the medium. Serial tenfold dilutions were then made in Tween 80 albumin medium. From appropriate dilutions volumes of $0.2 \mathrm{ml}$. were inoculated on to the surface of the tubes of Herrold's medium with dropping pipettes. A typical arrangement of an experiment is shown in Table 1. The tubes were kept in the incubator till the surface of the medium was dry and were then plugged with waxed corks. After 6 weeks' incubation at $37^{\circ}$ the number of colonies was counted in dilutions where up to 20 colonies appeared in each tube and a curve was obtained relating surviving organisms to streptomycin concentration.

Isolated colonies were then picked off into tubes of Tween 80 albumin medium. Usually, thirteen colonies were sampled from each streptomycin concentration, but in some of the experiments there was only a smaller number of separate colonies available in the tubes containing the higher concentrations of streptomycin. Strains were subcultured into a second set of tubes of Tween 80 albumin medium and, after 10 days' incubation, sensitivity tests to streptomycin were performed in the same medium by the method recommended by the Medical Research Council (1948) in which an inoculum of $0.02 \mathrm{ml}$. is used, and the tests are read after 10 days' incubation. 


\section{RESULTS}

Experiments with the strain $\mathrm{Pa}$ were set up on two occasions, and those with Pr and H37 Rv on one occasion each. The results of the sensitivity tests on the strains obtained by picking off colonies are given in Table $2 a-d$. With the strain $\mathrm{Pa}$ an equal number of colonies was sampled from each streptomycin concentration in Herrold's medium. The numbers of strains inhibited by the same concentration of streptomycin in the liquid medium sensitivity tests were then added up over the whole range of concentrations in the Herrold's medium. These totals, plotted against the inhibitory concentrations in the sensitivity tests, are shown in Fig. 1. Where the number of colonies sampled was not equal (strains $\mathrm{Pr}$ and $\mathrm{H37} \mathrm{Rv}$ ) the figures are adjusted so that equal weight is given to the sample from each streptomycin concentration in Herrold's medium. Thus, if ten colonies were sampled from one concentration and thirteen from another the numbers of tests from the latter would be multiplied by 10/13 and the totals added up as before. Since the streptomycin concentrations in

Table 2. The distribution of streptomycin resistance determined in Treen 80 albumin medium, of cultures from colonies growing in various concentrations of streptomycin in Herrold's medium

(a) Strain Pa, Exp. 1.

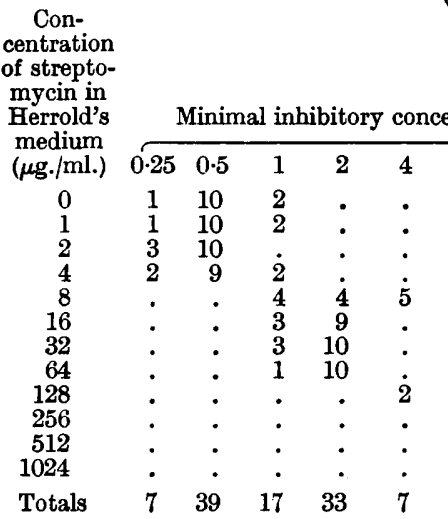

Concentration of streptomycin in Herrold's
medium medium

\begin{tabular}{|c|c|c|c|c|c|c|c|c|c|c|c|c|c|c|c|}
\hline$(\mu \mathrm{g} . / \mathrm{ml})$. & $0 \cdot 25$ & 0.5 & 1 & 2 & \pm & 8 & 16 & 32 & 64 & 128 & 256 & 512 & 1024 & 2048 & $>2048$ \\
\hline 0 & & 7 & 6 & - & - & - & - & - & - & - & - & - & . & - & - \\
\hline 2 & 2 & 8 & 3 & . & . & . & . & . & . & . & . & . & . & . & . \\
\hline 4 & 2 & 9 & 2 & & & . & . & . & . & . & . & . & . & . & . \\
\hline 8 & - & . & 3 & 9 & 1 & . & . & . & . & . & . & . & . & . & . \\
\hline 16 & . & . & 2 & 10 & 1 & . & . & . & . & . & . & - & . & - & - \\
\hline 32 & . & . & & 13 & & . & & & . & . & . & . & . & . & . \\
\hline 64 & . & . & 2 & 7 & 2 & - & 1 & 1 & 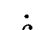 & - & - & - & - & - & $\dot{x}$ \\
\hline 128 & . & . & . & . & . & . & $\dot{0}$ & 2 & 6 & . & i & . & . & . & 5 \\
\hline 256 & - & - & - & - & - & - & 2 & 3 & 2 & - & 1 & - & - & $i$ & 5 \\
\hline 512 & - & - & - & - & - & - & & 1 & 2 & . & 1 & - & & 1 & 8 \\
\hline 1024 & - & . & . & - & • & . & 1 & 1 & . & . & . & . & 1 & - & 10 \\
\hline Totals & 4 & 24 & 18 & 39 & 4 & 0 & 4 & 8 & 10 & 0 & 2 & 0 & 1 & 1 & 28 \\
\hline
\end{tabular}

(b) Strain Pa, Exp. 2. 
Table 2 (cont.)

\begin{tabular}{|c|c|c|c|c|c|c|c|c|c|c|c|c|c|c|c|}
\hline \multirow{2}{*}{$\begin{array}{c}\text { Con- } \\
\text { centration } \\
\text { of strepto- } \\
\text { mycin in } \\
\text { Herrold's } \\
\text { medium } \\
(\mu \mathrm{g} . / \mathrm{ml} .)\end{array}$} & \multicolumn{15}{|c|}{ Minimal inhibitory concentration of streptomycin in sensitivity tests $(\mu \mathrm{g} . / \mathrm{ml})}$. \\
\hline & 0.25 & 0.5 & 1 & 2 & 4 & 8 & 16 & 32 & 64 & 128 & 256 & 512 & 1024 & 2048 & $>204$ \\
\hline $\begin{array}{r}0 \\
1 \\
2 \\
4 \\
8 \\
16 \\
32 \\
64 \\
128 \\
256 \\
512 \\
1024\end{array}$ & $\begin{array}{r}12 \\
12 \\
8 \\
5 \\
: \\
: \\
: \\
: \\
:\end{array}$ & $\begin{array}{l}1 \\
1 \\
4 \\
3 \\
: \\
: \\
: \\
: \\
:\end{array}$ & $\begin{array}{l}\dot{ } \\
\text { i } \\
5 \\
3 \\
6 \\
2 \\
\dot{.} \\
\dot{ } \\
.\end{array}$ & $\begin{array}{l}\dot{ } \\
\dot{ } \\
\dot{9} \\
4 \\
2 \\
\dot{.} \\
\dot{.}\end{array}$ & $\begin{array}{l}\dot{ } \\
\dot{2} \\
\dot{2} \\
\dot{ } \\
\dot{ }\end{array}$ & $\begin{array}{l}\dot{ } \\
\dot{.} \\
\text { i } \\
\text { i } \\
. \\
.\end{array}$ & $\begin{array}{l}\dot{ } \\
: \\
\dot{1} \\
\dot{1} \\
\dot{1} \\
\dot{0}\end{array}$ & $\begin{array}{l}: \\
\dot{5} \\
\dot{5} \\
\dot{3} \\
1 \\
1 \\
1 \\
\dot{.}\end{array}$ & $\begin{array}{l}\dot{ } \\
\dot{5} \\
\dot{1} \\
\dot{1} \\
1 \\
1 \\
\dot{ } \\
\dot{.}\end{array}$ & $\begin{array}{l}: \\
: \\
: \\
: \\
\mathrm{i} \\
2 \\
1 \\
.\end{array}$ & $\begin{array}{l}: \\
: \\
: \\
: \\
:\end{array}$ & $\begin{array}{l}: \\
: \\
: \\
: \\
:\end{array}$ & $\begin{array}{l}: \\
: \\
: \\
: \\
:\end{array}$ & $\begin{array}{l}: \\
\dot{1} \\
\dot{ } \\
\dot{2} \\
\dot{2}\end{array}$ & $\begin{array}{l}\dot{:} \\
\dot{5} \\
\dot{5} \\
\dot{4} \\
4 \\
4 \\
1 \\
4 \\
2\end{array}$ \\
\hline Totals & 37 & 9 & 17 & 15 & 2 & 2 & 2 & 6 & 3 & 4 & 0 & 0 & 0 & 3 & 19 \\
\hline $\begin{array}{c}\text { Adjusted } \\
\text { totals* }\end{array}$ & 11.38 & 2.91 & $5 \cdot 31$ & $4 \cdot 62$ & 0.62 & 0.95 & 1.18 & $2 \cdot 86$ & 1.52 & $2 \cdot 97$ & 0 & 0 & 0 & $2 \cdot 38$ & 11.81 \\
\hline
\end{tabular}

\author{
(c) Strain Pr.
}

(d) Strain H37 Rv.

Con-

centration

of strepto-

mycin in

Herrold's
medium

Minimal inhibitory concentration of streptomycin in sensitivity tests $(\mu \mathrm{g} . / \mathrm{ml}$.)

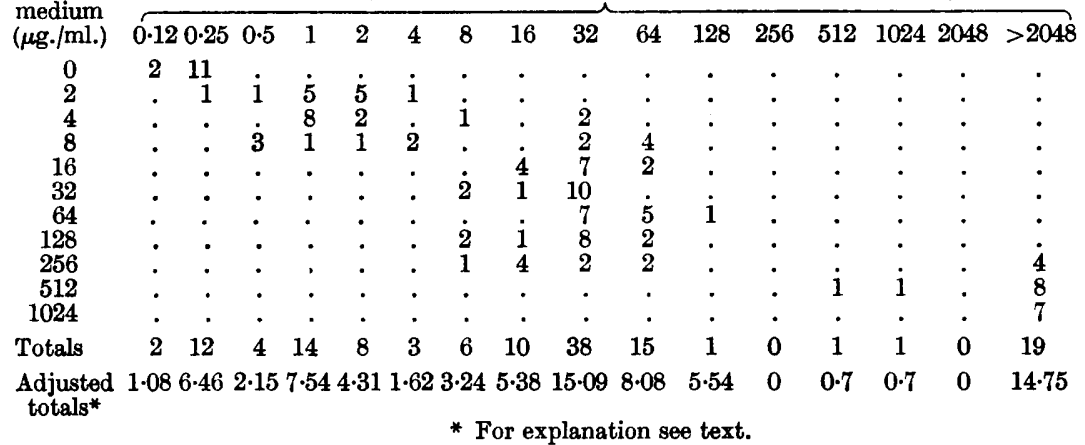

No. colonies sampled 13 13
13
13
13
13
13
13
13
10
7

Herrold's medium are equally spaced, peaks in the curves in Fig. 1 indicate a tendency for strains to segregate into groups with characteristic levels of resistance. It is clear that there are four such peaks. The first corresponds to sensitive strains, the second to slightly resistant strains, the third to moderately resistant strains and the fourth to highly resistant strains capable of growth in $2048 \mu \mathrm{g} . / \mathrm{ml}$.

In Fig. 1 the results of a number of sensitivity tests by the same method on strains derived from patients under treatment with streptomycin are also plotted. One part of the curve is derived from the tests on 192 strains isolated before treatment (Medical Research Council, 1948). The other part is derived from tests on 432 strains obtained from patients during treatment and after a strain at least four times less sensitive than the standard strain $\mathbf{H 3 7} \mathbf{R v}$ had been obtained, i.e. after some degree of resistance to streptomycin had appeared 
in the strains isolated. The results on 398 of these strains were published previously (Mitchison, 1949), but they were then expressed in terms of relative sensitivity to the standard strain. These data again show the presence of four peaks corresponding to those obtained in this paper.

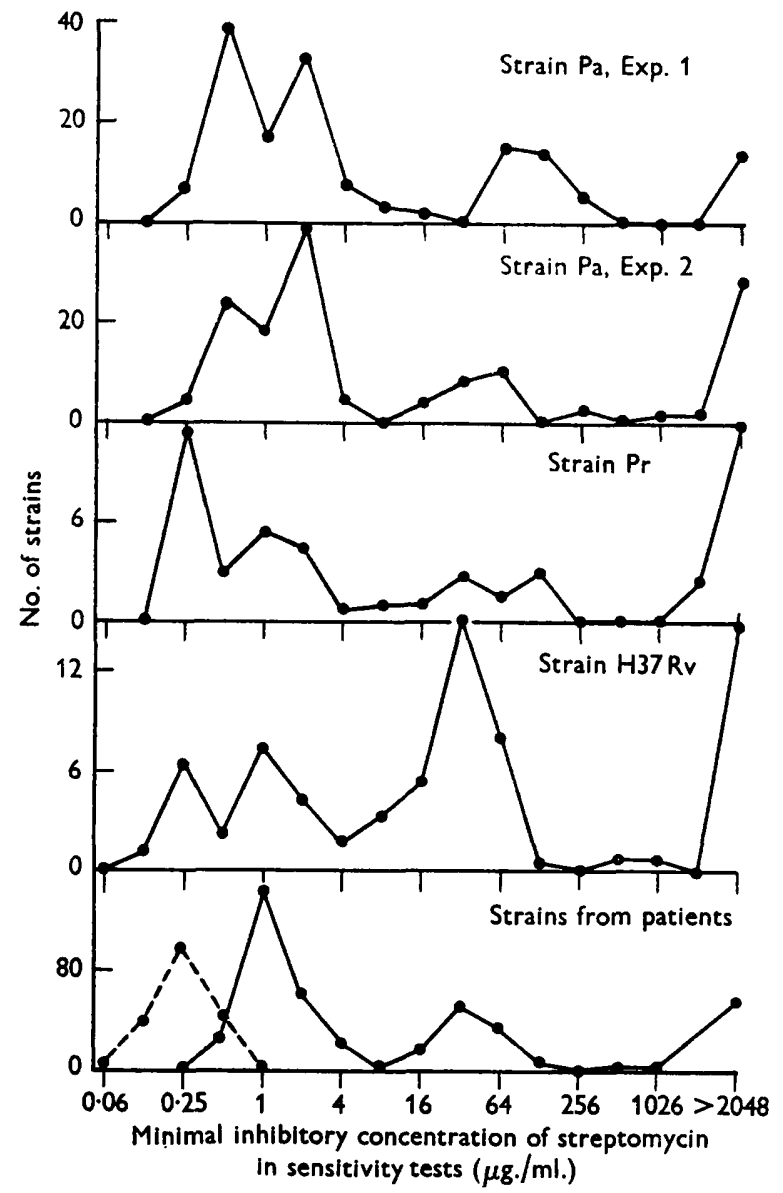

Fig. 1. The distribution of substrains of M. tuberculosis derived from Herrold's medium with respect to the minimal inhibitory concentration of streptomycin in the liquid medium sensitivity test. The values for Strains $\mathrm{Pr}$ and $\mathrm{H} 37 \mathrm{Rv}$ have been adjusted as described in the text, to allow for uneven sampling of the colonies on Herrold's medium. The strains from patients include $192(---)$ obtained before treatment with streptomycin, and 432 -) from patients yielding streptomycin-resistant strains.

The curves relating surviving organisms to streptomycin concentrations (survivor curves) are given in Fig. 2. These curves may be interpreted as a series of steps, corresponding to colony counts of sensitive, slightly resistant, moderately resistant and highly resistant strains. As the streptomycin concentration increases, there is first a sharp fall in the number of survivors accompanied by a disappearance of sensitive strains. There is then a relatively flat portion of curve, and slightly resistant strains were obtained from colonies 
in these tubes. This is followed by another fall in numbers and a second levelling off. Colonies picked from these concentrations usually yielded a mixture of moderately and highly resistant strains. There is considerable variation from experiment to experiment in the concentration of streptomycin in the Herrold's

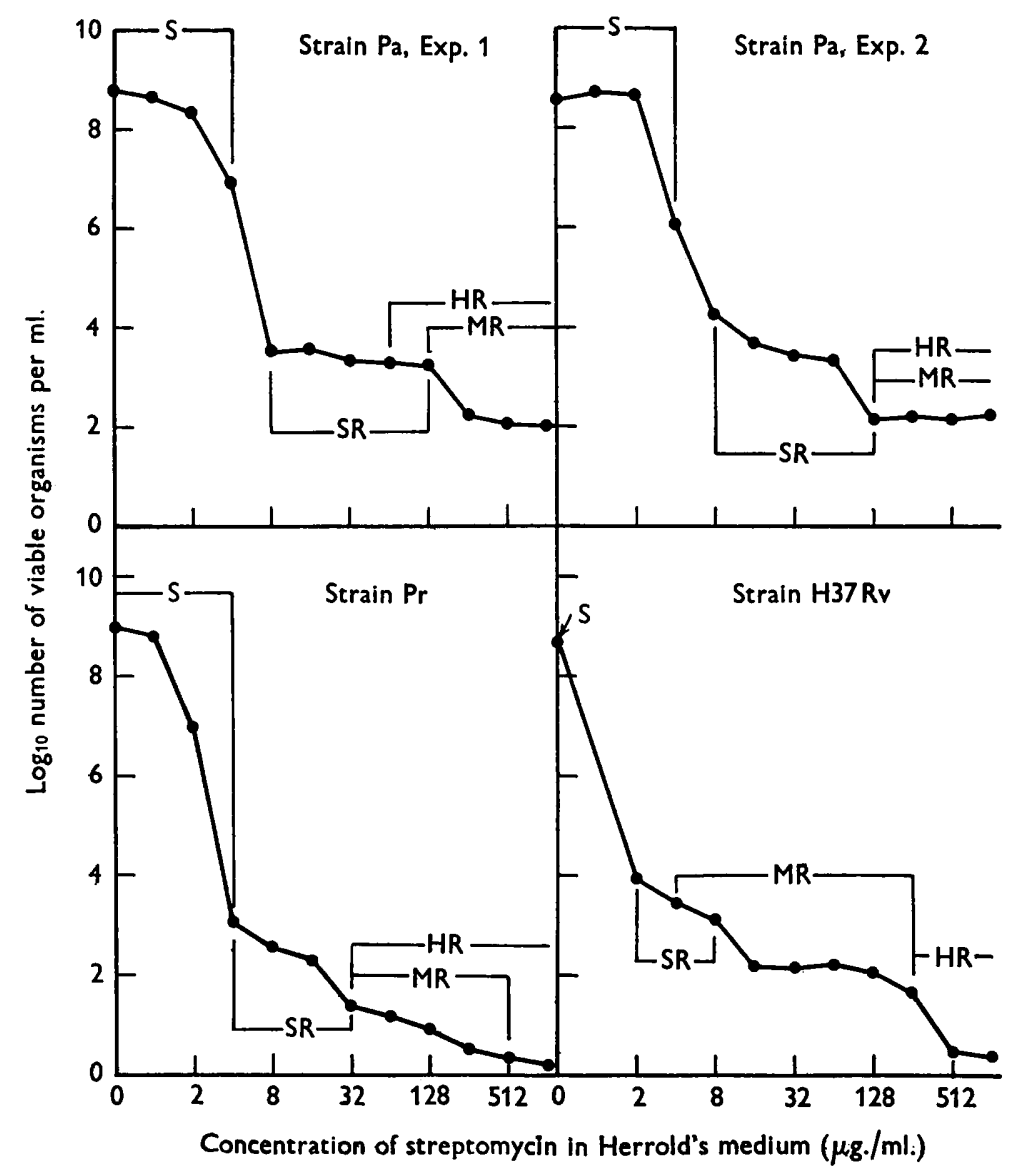

Fig. 2. Survivor curves of $M$. tuberculosis on streptomycin-containing media. $\log _{10}$ number of surviving organisms in different concentration of streptomycin in Herrold's medium. The substrains derived from Herrold's medium were sensitive over the streptomycin concentrations marked $\mathrm{S}$, slightly resistant over the streptomycin concentrations marked SR, moderately resistant over the streptomycin concentrations marked MR and highly resistant over the streptomycin concentrations marked HR.

medium which allows growth of colonies yielding strains of any particular level of resistance. In only one experiment, with $\mathrm{H3} \mathbf{R v}$, were the higher concentrations sufficient to prevent the growth of moderately resistant strains. Medium containing 512 and $1024 \mu \mathrm{g} . / \mathrm{ml}$. yielded highly resistant strains only and it will be seen that again there is another slight fall in surviving organisms between the tubes containing 256 and $512 \mu \mathrm{g} . / \mathrm{ml}$., but that approximately the same numbers survive in 1024 as in $512 \mu \mathrm{g} . / \mathrm{ml}$. 


\section{DISCUSSION}

Cultures of tubercle bacilli in Tween 80 albumin medium, although growing in a macroscopically dispersed manner, contain a mixture of separated bacilli and small clumps. The viable counts of these strains, when performed in the absence of streptomycin, will therefore tend to be an underestimate of the number of bacilli present, since some of the colonies will be derived from clumps rather than individual bacilli. However, in the presence of streptomycin only a small fraction of the bacilli are capable of growth and the error introduced by clumping becomes insignificant. For this reason the ratio between sensitive and resistant organisms derived from the survivor curves is likely to be too low, but the ratio between different types of resistant organisms is more likely to be accurate. Bearing this in mind, one can say that, out of a total population of $0 \cdot 4-1 \cdot 0 \times 10^{9}$ organisms in each ml., somewhere about 100 are slightly resistant, 100 are moderately resistant and 2 are highly resistant. The estimate for the highly resistant variants agrees well with that of Yegian \& Vanderlinde. These authors found that colonies growing on medium containing $100 \mu \mathrm{g}$. streptomycin $/ \mathrm{ml}$. yielded strains capable of growth on $1000 \mu \mathrm{g} . / \mathrm{ml}$., whereas, in the present work, most of the colonies derived from Herrold's medium containing 64 or $128 \mu \mathrm{g}$. $/ \mathrm{ml}$. were moderately resistant and, therefore, inhibited by $1000 \mu \mathrm{g} . / \mathrm{ml}$. This might be due to the presence of Tween 80 in Yegian \& Vanderlinde's solid medium which increases the apparent activity of a number of antibiotics including streptomycin (Youmans \& Youmans, 1948) and would probably inhibit moderately resistant strains in the presence of $100 \mu \mathrm{g}$. $/ \mathrm{ml}$.

The segregation of streptomycin resistant variants into three groups suggests that there are at least three separate biochemical processes which are interfered with by streptomycin. It is possible that the slightly resistant strains have developed an alternative pathway for one of these processes, but not for the other two, whereas the moderately resistant strains have alternative pathways for two of the processes and the highly resistant strains for all three. It is also possible that the resistance of the slightly resistant and moderately resistant organisms is due to a decreased permeability of the cells as has been suggested for penicillin resistance in yeasts by Maas \& Johnson (1949), or to the production of a resistant enzyme as Gots \& Sevag (1948) report for sulphonamide resistance.

Oginsky, Smith \& Umbreit (1949) consider that streptomycin prevents the formation of an oxaloacetate-pyruvate condensation product. They worked with Bacterium coli strains and with tissue homogenates. Highly resistant variants of the same Bact. coli strains were unable to affect this condensation. It would be interesting to know whether similar results could be obtained with sensitive and highly resistant tubercle bacilli, and whether the slightly and moderately resistant strains are capable of effecting the condensation.

This work was carried out during the receipt of a grant from the Medical Research Council, and with the technical assistance of Mr D. J. Rose. 


\section{REFERENCES}

Gots, J. S. \& Sevag, M. G. (1948). Enzymatic studies on the mechanism of resistance of pneumococci to drugs. III. Experimental results indicating alteration in enzyme proteins associated with the development of resistance to drugs. J. Bact. 56,737 .

MaAs, E. A. \& Johnson, M. J. (1949). Penicillin uptake by bacterial cells. J. Bact. $57,415$.

Meads, M. \& Haslam, N. H. (1949). Quantitative studies on the origin and characteristics of streptomycin-fast variants of Klebsielle pneumoniae. J.Immunol. 63, 1 .

Medical Research Council (1948). Specific laboratory tests in streptomycin therapy of tuberculosis. Lancet, ii, 862.

Mrtchison, D. A. (1949). Tests for streptomycin sensitivity of tubercle bacilli in Tween 80 albumin liquid medium. Lancet, ii, 604 .

Oginsky, E. L., Smith, P. H. \& Umbreit, W. W. (1949). The action of streptomycin. 1. The nature of the reaction inhibited. J. Bact. 58, 747 .

Pyle, M. M. (1947). Relative numbers of resistant tubercle bacilli in sputa of patients treated with streptomycin. Proc. Mayo Clin. 22, 465.

Stamp, Lord (1947). The preservation of bacteria by drying. J. gen. Microbiol. 1, 251.

Vennesland, K., Ebert, R. \& Bloch, R. (1947). The demonstration of naturally occurring streptomycin-resistant variants in the human strain of tubercle bacillus H $37 \mathrm{Rv}$. Science, $106,476$.

Yegian, D. \& Vanderlinde, R. J. (1948). A quantitative analysis of the resistance of mycobacteria to streptomycin. J. Bact. 56, 177.

Youmans, A. S. \& Youmans, G. P. (1948). Effect of 'Tween 80' in vitro on bacteriostatic activity of 20 compounds for Myco. tuberculosis. J. Bact. 52, 245.

(Received 8 March 1951) 